\title{
Analysis of pain indicators in a tertiary care hospital: study based on electronic clinical histories
}

Martínez S. , Trillo L. Garcia J., Montes A.

Parc de Salut MAR, Dept of Anaesthesiology, Intensive Care \& Pain Unit. Barcelona, Spain

\section{Background and Goal of Study}

Electronic clinical histories (ECHs) now used in most hospitals provide data for analysis of pain indicators. ${ }^{1}$ We aimed to compare pain intensity in patients treated on medical and surgical wards.

\section{Materials and methods}

Pain indicators for all patients admitted to the hospital's medical and surgical wards from July 2015 to June 2016 were analyzed. The indicators gathered from the ECHs were the percentages of patients reporting pain assessed as $>3$ (moderate) or $>7$ (severe) on a verbal numerical rating scale (NRS) once or twice consecutively. We used Pearson's $X^{2}$ test of independence to compare data for each quarter between medical and surgical wards.

\section{Results and Discussion}

Table 1 compares pain indicators reported on medical and surgical wards in each quarter. Significantly more patients on medical wards than surgical wards $(p<0.05)$ reported severe pain both once and twice consecutively in all quarters. The results for moderate pain reported once and twice consecutively by medical ward patients were better in some quarters, however. The incidence of moderate pain reported twice consecutively still exceeded the $5 \%$ recommended as a standard of care by some scientific associations. ${ }^{2}$

\section{Table 1. Pain on medical and surgical wards between July 2015 and June 2016}

\begin{tabular}{|l|c|c|c|c|c|c|c|c|}
\hline & \multicolumn{2}{|c|}{ JUL-SEP 2015} & \multicolumn{2}{c|}{ OCT-DEC 2015 } & \multicolumn{2}{c|}{ JAN-MAR 2016 } & \multicolumn{2}{c|}{ APR-JUN 2016} \\
\cline { 2 - 9 } & Medical & Surgical & Medical & Surgical & Medical & Surgical & Medical & Surgical \\
\hline No. of patients & 1873 & 2066 & 2334 & 2732 & 2314 & 2637 & 2281 & 2706 \\
\hline NRS > 3,\% & $39.4^{*}$ & $45.2^{*}$ & 40.9 & 42.8 & 40.4 & 42 & $38.5^{*}$ & $43.6^{*}$ \\
\hline NRS > 7,\% & $18.3^{*}$ & $14.7^{*}$ & $20.6^{*}$ & $14.4^{*}$ & $21.7^{*}$ & $14.1^{*}$ & $18.5^{*}$ & $14^{*}$ \\
\hline $\begin{array}{l}\text { NRS > 3 twice } \\
\text { consecutively, \% }\end{array}$ & 13.1 & 14.7 & 15.1 & 14.7 & $14.4^{*}$ & $16.8^{*}$ & 13.5 & 15.1 \\
\hline $\begin{array}{l}\text { NRS > 7 twice } \\
\text { consecutively, \% }\end{array}$ & $5.7^{*}$ & $2.1^{*}$ & $6.6^{*}$ & $1.8^{*}$ & $7.1^{*}$ & $3^{*}$ & $5.9^{*}$ & $2^{*}$ \\
\hline
\end{tabular}

*Statistically significant differences between medical and surgical wards $(p<0.05)$

\section{Conclusion}

Analysis of ECHs pain indicators demonstrated a higher incidence of intense pain on medical wards in our hospital. The incidence of two consecutive assessments revealing either moderate or severe pain on medical wards is higher than quality of care guidelines advise. These findings warrant analysis of the reasons for pain on these wards and the creation of protocols to assess and treat it.

\section{References}

1.Joint Commission on Accreditation of Healthcare Organizations. Pain assessment and management standards-Hospitals.

Comprehensive accreditation manual for hospitals. Handbook: Joint Commission on Accreditation of Healthcare Organizations, 2001

2.Raising the standard: A compendium of audit recipes.3ํe edit. The Royal College of Anesthetists 2012 